\title{
Estimating Lymphocyte Division and Death Rates from CFSE Data
}

\author{
Rob J. De Boer ${ }^{a, b, *}$, Vitaly V. Ganusova , Dejan Milutinovića, \\ Philip D. Hodgkinc ${ }^{\mathrm{c}}$, Alan S. Perelson ${ }^{\mathrm{b}, \mathrm{d}}$ \\ ${ }^{a}$ Theoretical Biology, Utrecht University, Padualaan 8, 3584 CH Utrecht, The \\ Netherlands \\ ${ }^{b}$ Santa Fe Institute, 1399 Hyde Park Road, Santa Fe, NM 87501, USA \\ ${ }^{c}$ The Walter and Eliza Hall Institute of Medical Research, Victoria, 3050, Australia \\ ${ }^{d}$ Theoretical Division, Los Alamos National Laboratory, Los Alamos, NM 87545, USA
}

Received: 21 September 2005 / Accepted: 3 February 2006 / Published online: 16 May 2006 (c) Society for Mathematical Biology 2006

\begin{abstract}
The division tracking dye, carboxyfluorescin diacetate succinimidyl ester (CFSE) is currently the most informative labeling technique for characterizing the division history of cells in the immune system. Gett and Hodgkin [Nat. Immunol. 1:239-244, 2000] have pioneered the quantitative analysis of CFSE data. We confirm and extend their data analysis approach using simple mathematical models. We employ the extended Gett and Hodgkin [Nat. Immunol. 1:239-244, 2000] method to estimate the time to first division, the fraction of cells recruited into division, the cell cycle time, and the average death rate from CFSE data on $\mathrm{T}$ cells stimulated under different concentrations of IL-2. The same data is also fitted with a simple mathematical model that we derived by reformulating the numerical model of Deenick et al. [J. Immunol. 170:4963-4972, 2003]. By a non-linear fitting procedure we estimate parameter values and confidence intervals to identify the parameters that are influenced by the IL-2 concentration. We obtain a significantly better fit to the data when we assume that the $\mathrm{T}$ cell death rate depends on the number of divisions cells have completed. We provide an outlook on future work that involves extending the Deenick et al. [J. Immunol. 170:49634972, 2003] model into the classical Smith-Martin model, and into a model with arbitrary probability distributions for death and division through subsequent divisions.
\end{abstract}

Keywords Cell division rate - Cell death rate - CFSE - Lymphocyte dynamics . IL-2

*Corresponding author.

E-mail address: r.j.deboer@bio.uu.nl 
Abbreviations: "anti-CD3 mAb" is a monoclonal antibody (mAb) triggering $\mathrm{T}$ cell activation via the $\mathrm{CD} 3$ molecule associated with $\mathrm{T}$ cell antigen receptors; interleukin-2 (IL-2) is a growth factor for T cells that is measured in "units/ml", where $1 \mathrm{unit} / \mathrm{ml}$ is the concentration required to give $50 \%$ of the maximum response in the HT2 bioassay (Mosmann et al., 1986); "thymidine" is radioactively labeled ${ }^{3} \mathrm{H}$-thymidine that is incorporated in the DNA of dividing cells; $\mathrm{BrdU}$ is the nucleotide analog 5-bromo-2'-deoxyuridine also incorporated in the DNA of dividing cells; CFSE is a color dye "carboxyfluorescin diacetate succinimidyl ester" which is taken up in the cytosol of cells, and which dilutes twofold when cells divides

\section{Introduction}

Carboxyfluorescin diacetate succinimidyl ester (CFSE) is an intracellular fluorescent dye that dilutes twofold when a cell divides (Lyons, 2000). The labeling of cells with CFSE is done in vitro, and labeled cells can be followed thereafter both in vitro and in vivo for long periods. Harvesting the cells and measuring CFSE intensity by flow cytometry generates profiles with maximally 7 or 8 peaks, each reflecting the number of divisions the cells have undergone. The limit of 7 or 8 divisions is caused by the dilution of the dye: after 8 divisions the CFSE intensity is $2^{8}$ fold lower than the original intensity, and is essentially equivalent to the autofluoresence of non-CFSE labeled cells. CFSE labeling is currently the most informative technique for characterizing the kinetics of cell division in the immune system.

A distinctive feature of CFSE data is that it reveals that cells in a population are spread over a number of division rounds revealing considerable heterogeneity in the behavior of cells. Gett and Hodgkin (2000) proposed an elegant and intuitive approach for analyzing CFSE data and interpreting the heterogeneity. The method, which we call the GH method, follows from experimental evidence that variability in the time to enter first division were primarily responsible for the heterogeneity. Thus, Gett and Hodgkin (2000) treated the first division as special and developed a graphical method to estimate the subsequent division rate.

To analyze CFSE data one first integrates the CFSE intensity under each peak, to measure the number of cells that have divided a given number of times (Hasbold et al., 1999). Gett and Hodgkin (2000) then normalize this data by the twofold expansion that is associated with each division. This has the immediate advantage of allowing one to see the distribution of cells from the original starting population in the various division numbers not confounded by their clonal expansion. CFSE data have frequently been misinterpreted by arguing that the large number of cells in the highest division number means that most cells have proceeded through this many divisions over the time of the experiment (Kaech and Ahmed, 2001; Van Stipdonk et al., 2001). Because each division involves a twofold expansion one expects more cells for the higher division numbers. Normalization reveals that the cells in the highest division number typically represent a small fraction of the starting population. Gett and Hodgkin (2000) analyzed the normalized distribution of the frequency of cells in each division number by fitting a 
normal distribution to the data, excluding the data corresponding to the division number zero, since some cells in this class may never divide. They then showed that the mean of the fitted normal distribution increased linearly in time, and argued that the slope represented the division rate. This seems a powerful notion, because intuitively one indeed expects the increase of the mean division number to reflect the division rate, whatever the precise kinetic mechanism by which cells divide. Here we analyze the validity of the GH method using simple mathematical models (De Boer and Perelson, 2005), and we employ the GH method to obtain initial estimates for the time to first division, the cell cycle time, and the death rate.

Deenick et al. (2003) extended the original GH method (Gett and Hodgkin, 2000) for analyzing CFSE data by manually fitting a six parameter model to the data. The model assumed that the time to first division fits a normal distribution, which is described by a mean and variance, and that subsequent divisions are deterministic and have a fixed length. The model included a death rate for non-divided cells, a death rate during division, and incorporated a fraction of the precursor cells that fail to divide. Recently Leon et al. (2004) generalized this model by rewriting it into a probabilistic form with an arbitrary probability distribution for the cell division time. Both models fitted well to a set of experiments in which T cells were stimulated in vitro with anti-CD3 monoclonal antibody $(\mathrm{mAb})$, and different concentrations of IL-2 (Deenick et al., 2003; Leon et al., 2004). Because both papers failed to provide confidence intervals on the parameter estimates, it is difficult to evaluate the uniqueness of the fit. In this paper we rewrite the Deenick et al. (2003) model into a single differential equation (ODE) for the first division cohort with parameters that depend on time, and an algebraic scaling equation for the subsequent cohorts. Fitting the new model to the same IL-2 data and bootstrapping the residuals (Efron and Tibshirani, 1986), we obtain estimates for the parameter values with confidence intervals.

Most of the earlier work on modeling CFSE dilution has been done with models resembling the Smith and Martin (1973) model with an explicit time delay for the S, G2, and M phases of the cell cycle (Nordon et al., 1999; Bernard et al., 2003; Pilyugin et al., 2003; De Boer and Perelson, 2005; Ganusov et al., 2005), or with other models allowing for an explicit time delay (Zhang et al., 2001; Deenick et al., 2003; Leon et al., 2004). CFSE data have also been interpreted by fitting the data to models that do not explicitly incorporate a minimum cell cycle length, i.e., autonomous ODE models where the times to division and death are exponentially distributed (Veiga-Fernandes et al., 2000; Revy et al., 2001). For cells that divide rapidly, we have recently shown that models with a delay like the classical Smith and Martin (1973) model or the above-mentioned Deenick et al. (2003) model, do a much better job at describing the dynamics of subpopulations of cells that have divided a given number of times than ODEs with exponentially distributed times to division (Pilyugin et al., 2003; De Boer and Perelson, 2005; Ganusov et al., 2005). The mean behavior of a population of cells can be modeled well by ODEs, but modeling the number of divisions a cell subpopulation has gone through may not be accurately represented when simple autonomous ODEs are used (De Boer and Perelson, 2005). In this paper we will use ODE models to illustrate the GH method of estimating the division time from the increase of the mean division number. For fitting we then use the Deenick et al. (2003) model with an 
explicit time delay to represent the cell cycle time of the second and subsequent divisions.

After confirming and extending the GH method by applying it to simple ODE models, we will fit CFSE data with the Deenick et al. (2003) model. Extending this model with a death rate that changes with the cell division number we obtain a significant improvement of the fit. For the extended model we determine which parameters vary with the concentration of IL-2 by determining their confidence intervals.

\section{Estimating slopes from autonomous ODEs}

Revy et al. (2001) proposed the following simple system of ODEs to analyze their CFSE data:

$$
\begin{aligned}
\frac{\mathrm{d} N_{0}}{\mathrm{~d} t} & =-(p+d) N_{0} \\
\frac{\mathrm{d} N_{i}}{\mathrm{~d} t} & =2 p N_{i-1}-(p+d) N_{i}, \quad i=1, \ldots, \infty
\end{aligned}
$$

where $N_{i}$ is the number of cells that have completed $i$ divisions, and $p$ and $d$ are the cell proliferation and death rates, respectively. The division number or division index is $i$. The solution of this system, with the initial condition $N_{0}(0)>0, N_{i}(0)=$ 0 for $i=1, \ldots, \infty$, is

$$
\begin{aligned}
& N_{0}(t)=N_{0}(0) \mathrm{e}^{-(p+d) t} \\
& N_{i}(t)=\frac{(2 p t)^{i}}{i !} N_{0}(t), \quad i=1, \ldots, \infty,
\end{aligned}
$$

which in practice remains low-dimensional because $N_{i}(t) \rightarrow 0$ when $i \rightarrow \infty$. The total number of cells, $N(t)=\sum_{0}^{\infty} N_{i}(t)=N_{0}(t) \mathrm{e}^{2 p t}=N_{0}(0) \mathrm{e}^{(p-d) t}$, changes exponentially with the net growth rate of the population $p-d$. The frequency distribution of cells over the division numbers, $F_{i}(t) \equiv N_{i}(t) / N(t)$, is given by a Poisson distribution

$$
F_{i}(t)=\frac{(2 p t)^{i}}{i !} \mathrm{e}^{-2 p t}, \quad i=0, \ldots, \infty
$$

Because this distribution only depends on the proliferation rate, $p$, it was used to estimate $p$ from CFSE data collected at various times after cells were placed in culture (Revy et al., 2001).

The GH method can be illustrated with this model by normalizing the number of cells in each division class by the corresponding degree of clonal expansion, i.e., the number of cells in division class $i$ is divided by $2^{i}$. This normalization is extremely useful and gives the distribution of cells from the starting population $N(0)$ over the various division classes. This is what Gett and Hodgkin (2000) call the precursor 
cohort distribution. When $n_{i}(t) \equiv N_{i}(t) / 2^{i}$, we obtain

$$
\begin{aligned}
& n_{0}(t)=N_{0}(t)=N_{0}(0) \mathrm{e}^{-(p+d) t} \quad \text { and } \\
& n_{i}(t)=\frac{(p t)^{i}}{i !} N_{0}(t), \quad \text { for } \quad i=1, \ldots, \infty .
\end{aligned}
$$

The total normalized cell number

$$
n(t) \equiv \sum_{0}^{\infty} n_{i}(t)=N_{0}(0) \mathrm{e}^{-d t}
$$

declines constantly with the death rate $d$, and the frequency distribution of cells in the different division classes is

$$
f_{i}(t) \equiv n_{i}(t) / n(t)=\frac{(p t)^{i}}{i !} \mathrm{e}^{-p t},
$$

which again is a Poisson distribution with mean and variance

$$
\mu(t) \equiv \sum_{i=0}^{\infty} i f_{i}(t)=p t \text { and } \sigma^{2}(t)=\sum_{i=0}^{\infty} i^{2} f_{i}(t)-\mu(t)^{2}=p t .
$$

Note that we obtain a Poisson precursor cohort distribution from this simple model having an exponential distribution of times to first division. For sufficiently large $t$ this Poisson distribution will resemble a normal distribution. Observing an apparently normal (or log-normal) precursor cohort plot (Gett and Hodgkin, 2000; Deenick et al., 2003) is therefore not enough to conclude that the time to first division is normally or log-normally distributed, i.e., one cannot infer the distribution of times to first division from the precursor cohort distribution.

Whatever the precise mechanisms underlying the time to first division, these results allow one to obtain an initial guess for three important parameters. First, the death rate $d$ can be estimated from the slope $\mathrm{e}^{-d t}$ by which the normalized total cell number decreases, see Eq. (5). Second, the division rate is given by the slope of the linear increase of $\mu(t)$, see Eq. (7). Finally, by adding a time delay to the model (Bernard et al., 2003; De Boer and Perelson, 2005), one can allow for the extra time required for the first division, and estimate the time to first division from the time at which the mean division number $\mu(t)=1$; see also (Gett and Hodgkin, 2000). Although we have included non-divided cells, and we have computed the mean directly from the Poisson distribution, rather than from a fitted normal distribution as in the GH method, the latter two conclusions mathematically confirm the estimation procedure proposed by Gett and Hodgkin (2000). We have added the simple estimation of a death rate to the procedure (De Boer and Perelson, 2005), and have shown elsewhere that in other models the linear increase of the mean division number with time, i.e., Eq. (7), is approached after an initial transient only (De Boer and Perelson, 2005; Ganusov et al., 2005). 
Finally, there is an interesting difference between the mean of this Poisson distribution, and the mean of the normalized data when the non-divided cells are excluded (as was originally proposed by Gett and Hodgkin (2000)). First, extend the model with a parameter $\phi$ for the fraction of precursor cells that will ultimately divide. For the cells that will ultimately divide one can still use the solution of Eq. (4) when one replaces the initial condition $N(0)$ by $\phi N(0)$. For the cells that fail to divide one has $(1-\phi) N(0) \mathrm{e}^{-d t}$. The frequency distribution $f_{0}(t) \equiv\left(\phi n_{0}(t)+(1-\phi) N(0) \mathrm{e}^{-d t}\right) / n(t)$ and $f_{i}(t) \equiv \phi n_{i}(t) / n(t)$, for $i=1, \ldots, \infty$, where $n_{0}(t), n_{i}(t)$ and $n(t)$ are given by Eqs. (4) and (5), becomes ${ }^{1}$

$$
f_{0}(t)=\phi \mathrm{e}^{-p t}+1-\phi \quad \text { and } \quad f_{i}(t)=\phi \frac{(p t)^{i}}{i !} \mathrm{e}^{-p t}
$$

with mean

$$
\mu(t) \equiv \sum_{i=0}^{\infty} i f_{i}(t)=\phi p t
$$

Thus, the mean division number increases linearly in time but with slope $\mu(t)^{\prime}=$ $\phi p$, which would argue that one cannot simply estimate the division time from the slope. However, if one computes a mean excluding the non-divided cells by defining $\widehat{\mu}(t) \equiv \sum_{i=1}^{\infty} i f_{i}(t) / \sum_{i=1}^{\infty} f_{i}$, one obtains

$$
\widehat{\mu}(t)=\frac{p t}{1-\mathrm{e}^{-p t}},
$$

which equals one when $t \rightarrow 0$, and which approaches a slope $\widehat{\mu}(t)^{\prime}=p$ after an initial transient corresponding to a few cell cycles (De Boer and Perelson, 2005). Comparing the slopes of both means for the same data set, one therefore obtains information about the fraction of precursor cells that are recruited into division, $\phi$. If $\mu(t)$ and $\widehat{\mu}(t)$ have a similar slope, one expects $\phi \simeq 1$, and one could have a first guess for the cell cycle time $1 / p$ from the slope of either of the two means. If the slopes are different, the ratio of the means or of their slopes provides a first guess for $\phi$ because asymptotically $\mu(t) / \widehat{\mu}(t)=\mu(t)^{\prime} / \widehat{\mu}(t)^{\prime}=\phi$. The same result can also be obtained in a more general way from the definition of the two means. Since one can rewrite

$$
\widehat{\mu}(t) \equiv \frac{\sum_{i=1}^{\infty} i f_{i}}{\sum_{i=1}^{\infty} f_{i}} \quad \text { as } \quad \widehat{\mu}(t)=\frac{\sum_{i=0}^{\infty} i f_{i}}{\sum_{i=0}^{\infty} f_{i}-f_{0}}=\frac{\mu(t)}{1-f_{0}(t)},
$$

where $\left(1-f_{0}(t)\right)$ is the fraction of cells triggered into division at time $t$. Using Eq. (8) one can see that $1-f_{0}(t)$ should asymptotically approach $\phi$.

Summarizing, plotting the two means, $\mu(t)$ and $\widehat{\mu}(t)$, and the total normalized cell numbers, $n(t)$, and fitting these with linear regression, one obtains an initial

\footnotetext{
${ }^{1}$ The total number of cells $n(t)$ does not change since the number that ultimately divide is $\phi N_{0}(0) \mathrm{e}^{-d t}$ and the number that do not divide is $(1-\phi) N_{0}(0) \mathrm{e}^{-d t}$.
} 
guess for the time to first division, the cell cycle time, the fraction of precursor cells that will divide, the loss of undivided cells, and an overall cell death rate. These estimates should be treated as a simple initial guess, mainly because other models, as discussed in the Introduction (Pilyugin et al., 2003; Ganusov et al., 2005), give rise to other interpretations of the data. They may provide good starting points for fitting with a more complicated model.

\subsection{Estimating five slopes from CFSE data}

We illustrate the method of estimating cell kinetic parameters from CFSE data by applying the methods discussed above to data on the dynamics of $\mathrm{CD}^{+} \mathrm{T}$ cells stimulated in vitro with anti-CD3 $\mathrm{mAb}$ in the presence of four different concentrations of IL-2 (Deenick et al., 2003). The two highest concentrations of IL-2 (5 and $50 \mathrm{U} / \mathrm{ml}$ ) are at a saturating level, and the $\mathrm{T}$ cells undergo a rapid proliferation cascade. In the absence of IL-2 cells hardly proliferate (Deenick et al., 2003), and at a concentration of 1.25 or $2.5 \mathrm{U} / \mathrm{ml}$ of IL-2 the proliferation is intermediate (Deenick et al., 2003). The mean division numbers, and the population sizes of the T cell data are depicted in Fig. 1. The fairly linear increase in the two mean division numbers, $\mu(t)$ and $\widehat{\mu}(t)$, suggests that one can consider the parameters of the model to remain reasonably homogeneous over the entire experiment. The fact that the two means increase with a similar slope suggest that most precursor cells are triggered to divide (i.e., $\phi \simeq 1$ ). We have fitted the data in Fig. 1 by linear regression, and obtained an initial guess for the death rate $(d)$, the cell cycle time $\left(1 / \widehat{\mu}^{\prime}\right)$, the time to first division (solved from $\widehat{\mu}=1$ ), and the fraction of precursor cells $(\phi)$; see Table 1. An important advantage of this simple graphical analysis of CFSE data is that it can be used as a check for inconsistencies in the data. For instance, the fact that the normalized cell numbers are increased at $57 \mathrm{~h}$ over that seen at $48 \mathrm{~h}$, with an IL-2 concentration of $5 \mathrm{U} / \mathrm{ml}$ (Fig. 1d squares) suggests an experimental error in the estimated total cell numbers at this time point, because the normalized cell number can only decrease (see Eq. (5)).

The parameter estimates provided in Table 1 suggest that increasing the IL-2 concentration, decreases the cell cycle time, $1 / \widehat{\mu}^{\prime}$, and increases the fraction of precursors, $\phi$, that will ultimately divide. The death rate, $d$, and the time to first division, $\widehat{\mu}=1$, are less affected by the IL-2 concentration (Table 1). These observations are in reasonable agreement with the earlier analysis of the data with the semi-probabilistic model of (Deenick et al., 2003). The main exception is the division time, $1 / \widehat{\mu}^{\prime}$, which is approximately twofold longer in Table 1 than that estimated by Deenick et al. (2003) or by us in Table 2. This will be discussed further below. An IL-2 concentration of $1.25 \mathrm{U} / \mathrm{ml}$ is apparently not enough to sustain the proliferation cascade because the net growth rate is negative. The rate at which undivided cells disappear by division and death is fairly independent of the IL-2 concentration, and is only slightly higher than the overall death rate.

\section{The Deenick et al. (2003) model}

For these $\mathrm{T}$ cells that were polyclonally stimulated into a cascade of rapid cell divisions, Deenick et al. (2003) proposed that after their first division cells 
(a)

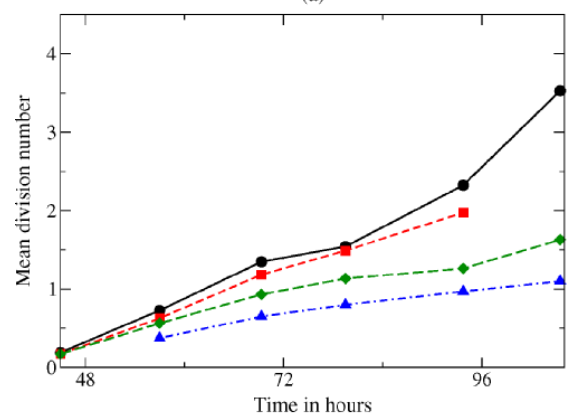

(c)

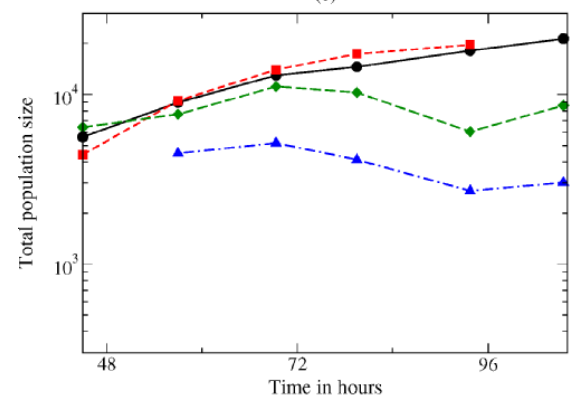

(b)

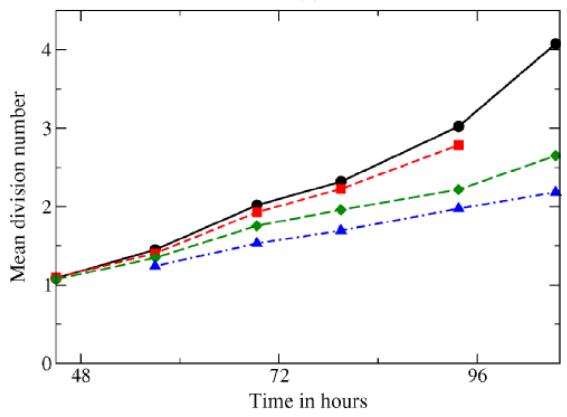

(d)

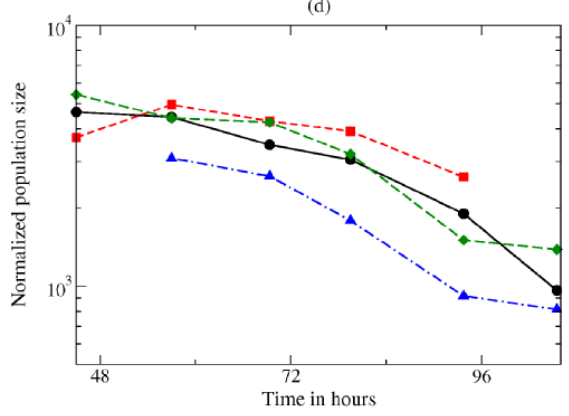

(e)

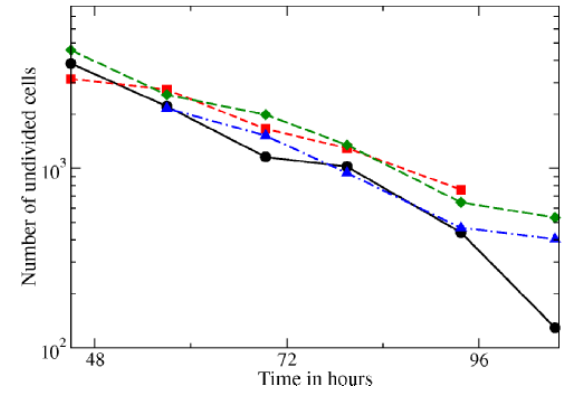

Fig. 1 Mean division numbers and population sizes of the T cell data. The symbols denote experimental data (Deenick et al., 2003) obtained at different concentrations of IL-2 (circles: $50 \mathrm{U} / \mathrm{ml}$, squares: $5 \mathrm{U} / \mathrm{ml}$, diamonds: $2.5 \mathrm{U} / \mathrm{ml}$, and triangles: $1.25 \mathrm{U} / \mathrm{ml}$ ). The five panels depict the mean division number $\mu$ (a) and $\widehat{\mu}$ (b), the total population size (c), the normalized population size (d), and the number of undivided cells (e).

proceed through subsequent divisions deterministically with a fixed cell cycle time $\Delta$ and death rate $d$. The main differences between the cells was assumed to be the time required to complete their first division, and this was modeled as a normal or log-normal distribution (Deenick et al., 2003). A plausible explanation for the differences between cells might be that they express different densities of the receptors involved in anti-CD3 stimulation, and that receptor densities typically obey log-normal distributions (Deenick et al., 2003).

Defining $R(t)$ as the density of cells completing their first division at time $t$ one can write the Deenick et al. (2003) model as one non-autonomous ODE and a set 
Table 1 Parameter estimates obtained from the slopes and intercepts of the regression lines fitted to the data in Fig. 1, excluding the initial time point.

\begin{tabular}{lllllll}
\hline $\mathrm{IL}-2(\mathrm{U} / \mathrm{ml})$ & $r(1 / \mathrm{h})$ & $d_{0}(1 / \mathrm{h})$ & $d(1 / \mathrm{h})$ & $1 / \widehat{\mu}^{\prime}(\mathrm{h})$ & $\widehat{\mu}=1(\mathrm{~h})$ & $\phi$ \\
\hline 0.125 & -0.011 & 0.042 & 0.031 & 52.1 & 43.2 & 0.76 \\
2.5 & 0.001 & 0.036 & 0.025 & 39.5 & 42.1 & 0.89 \\
5 & 0.030 & 0.030 & 0.008 & 28.5 & 43.6 & 1.0 \\
50 & 0.021 & 0.052 & 0.025 & 21.1 & 46.9 & 1.0 \\
\hline
\end{tabular}

Note. $r$ is the growth rate as estimated by linear regression of the data in Fig. $1 \mathrm{c}, d_{0}$ is the rate at which undivided cells disappear as estimated by linear regression from the data in Fig. 1e, $d$ is the death rate estimated by linear regression from the data in Fig. $1 \mathrm{~d}, 1 / \widehat{\mu}^{\prime}$ reflects the cell cycle time estimated from the linear increase in the mean division number (excluding the non-divided cells), $\widehat{\mu}=1$ estimates the time to first division from the time at which this linear regression line intersects $\widehat{\mu}=1$ (Gett and Hodgkin, 2000), and $\phi$ is estimated from the ratio of the slopes of the two mean division numbers, i.e., $\phi=\mu^{\prime} / \widehat{\mu}^{\prime}$.

of algebraic scaling equations, i.e., as

$$
\begin{aligned}
\frac{\mathrm{d} N_{1}(t)}{\mathrm{d} t} & =R(t)-R(t-\Delta) \mathrm{e}^{-d \Delta}-d N_{1}(t), \quad N_{1}(0)=0, \\
N_{i}(t) & =\left[2 \mathrm{e}^{-d \Delta}\right]^{i-1} N_{1}(t-[i-1] \Delta), \quad N_{i}(0)=0, \quad i=2, \ldots, \infty .
\end{aligned}
$$

We will call this the DGH model. The middle term in the ODE describes the cells that have not died, and thus have successfully completed their second division, and proceed to the next division stage. The algebraic equation is based on the fact that the cells in the later divisions are those that complete, do not die, and take a time $\Delta$ to complete each division. The shape of the solution of the later divisions is identical to the first one, except for a shift in time and a scaling for the total area under the curve by the death rate. In the Appendix we give the log-normal distribution, $R(t)$, with four parameters $\left(\mu, \sigma, \Delta_{0}\right.$, and $\left.C\right)$ that was used to fit the data, and we show how Eq. (12) can be derived. Employing this simple model we ignore the data on the non-divided cells, which are a mixture of dying cells, cells that will ultimately divide, and cells that will never divide.

This DGH model was fitted by minimizing the sum of the squared residuals using the FindMinimum routine in Mathematica to the four data sets obtained with different concentrations of IL-2. To avoid problems with the autofluoresence of unlabeled cells overlapping with $\mathrm{CFSE}^{+} \mathrm{T}$ cells that have divided more than six times (Hasbold et al., 1999; Milutinović, 2004), we combine the data and the model predictions of the 7 th and 8 th division numbers into a lumped category that we call $6+$ (Fig. 2). Comparing the model predictions with the CFSE profiles over the different division numbers suggests that the fits are very reasonable (Fig. 2). Depicting the same fits as a function of time for the various division cohorts, $N_{i}(t)$, shows that the fits for low IL-2 concentrations are actually rather poor (Fig. $3)$. Take for example the highest peak for the IL-2 concentration of $1.25 \mathrm{U} / \mathrm{ml}$, which in the data (red triangles) occurs for cells that have divided twice, and in the model (short-dashed line) occurs for cells that have divided once (Fig. 3). Similarly, divisions $1-3$ with an IL-2 concentration of $2.5 \mathrm{U} / \mathrm{ml}$ are rather poorly described by the fits. Note that all distributions of the different cohorts have the same shape except for a scaling of the area under the curve due to cell death: the form of 


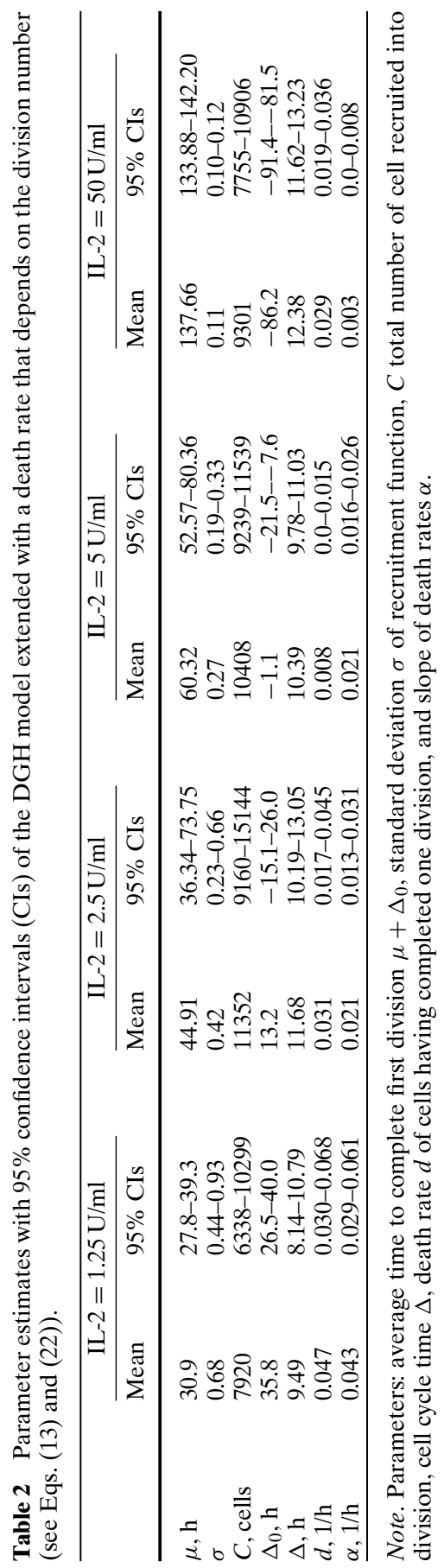


(a)

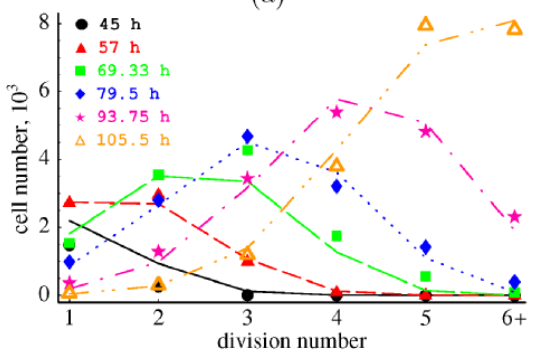

(c)

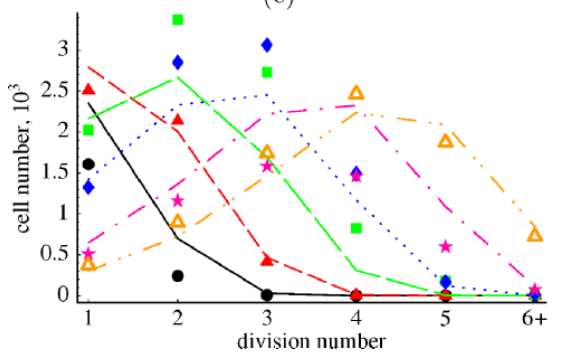

(b)

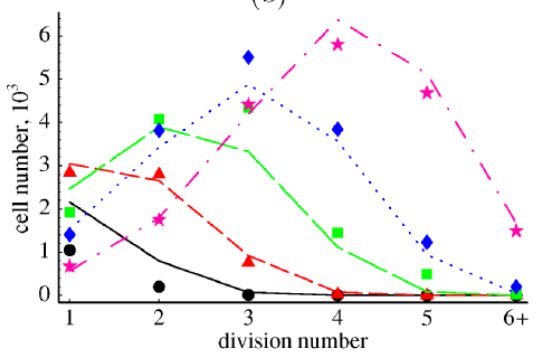

(d)

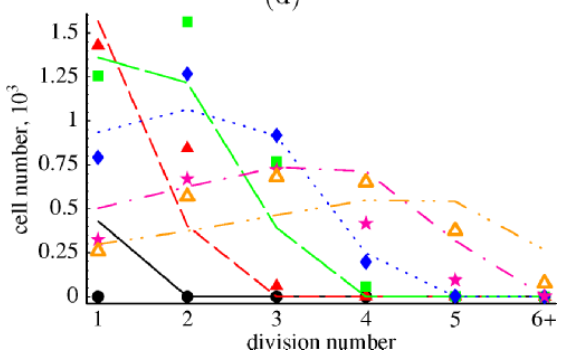

Fig. 2 CFSE profiles of the Deenick et al. (2003) model fitted to CFSE data for IL-2 concen-

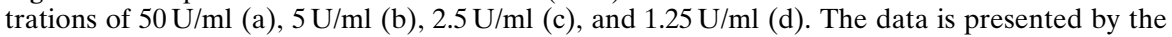
symbols and the fit of the model as lines. Time points: $45 \mathrm{~h}$ (solid line with circles), $57 \mathrm{~h}$ (shortdashed line with triangles), $70 \mathrm{~h}$ (long-dashed line with squares), $80 \mathrm{~h}$ (dotted line with diamonds), $94 \mathrm{~h}$ (dash-dotted line with stars), and $105 \mathrm{~h}$ (double dash-dotted line with open triangles).

these curves is the integral of $R(t)$ over time (see Eq. (12)). Except for the lowest IL-2 concentration the model fits a fairly symmetric distribution, $R(t)$, which is in disagreement with the asymmetric recruitment functions found by thymidine incorporation (Deenick et al., 2003). Summarizing, the cohort plots of Fig. 3 allow for a more critical judgment of the quality of the fit, and suggest that the DGH model has to be extended in order to better explain the data.

\subsection{Heterogeneous model}

The fact that for the lowest concentration of IL-2 the third and later cohorts approach much lower peak values that the first two cohorts (Fig. 3), suggests that the division rate, and/or the death rate, change over time or with the number of divisions completed. The model of Eq. (12) was uniform in the sense that after the first division, all parameters remain independent of the division number and time. Data obtained after a short pulse with BrdU suggest that the cell cycle time, does not decrease with the division number, because the fraction of $\mathrm{BrdU}^{+}$cells is approximately independent of the division number (Deenick et al., 2003). We therefore extend the DGH model by incorporating a non-uniform death rate of cells. We assume that $d_{i}=d+\alpha(i-1)$, where $d$ is the death rate of the first cohort, and $\alpha$ is the slope with which the death rate changes with the division number. Writing the 
(a)

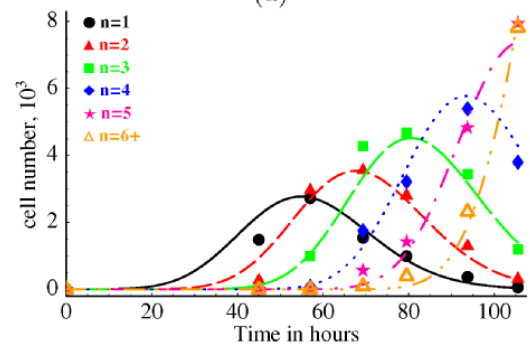

(c)

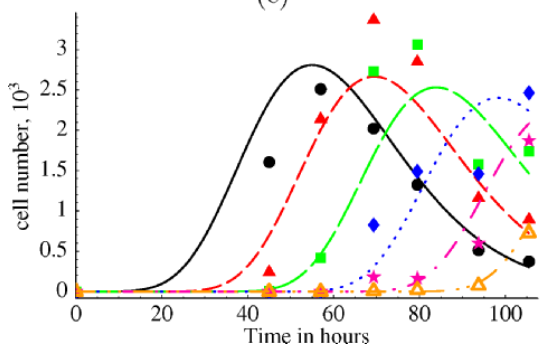

(b)

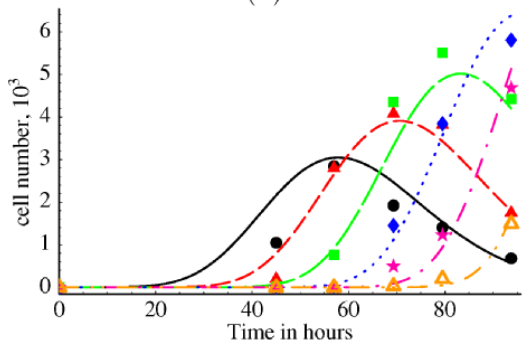

(d)

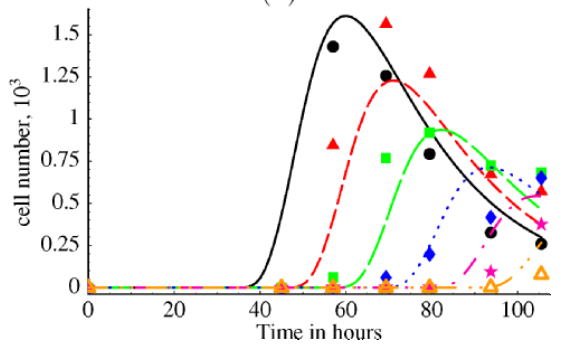

Fig. 3 The same fit of the Deenick et al. (2003) as shown in Fig. 2 shown as cell cohorts with the same division number. IL-2 concentrations of $50 \mathrm{U} / \mathrm{ml}$ (a), $5 \mathrm{U} / \mathrm{ml}$ (b), $2.5 \mathrm{U} / \mathrm{ml}$ (c), and $1.25 \mathrm{U} / \mathrm{ml}$ (d). The data is presented by the symbols and the fit of the model as lines. Starting from the cohort having completed one division (solid line with circles), two divisions (short-dashed line with triangles), three (long-dashed line with squares), four (dotted line with diamonds), five (dash-dotted line with stars), to six or more (double dash-dotted line with open triangles).

model as a system of ODEs, the model becomes

$$
\begin{aligned}
\frac{\mathrm{d} N_{1}}{\mathrm{~d} t}= & R(t)-R(t-\Delta) \mathrm{e}^{-d_{1} \Delta}-d_{1} N_{1}, \\
\frac{\mathrm{d} N_{i}}{\mathrm{~d} t}= & 2^{i-1} R(t-(i-1) \Delta) \mathrm{e}^{-\sum_{j=1}^{i-1} d_{j} \Delta} \\
& -2^{i-1} R(t-i \Delta) \mathrm{e}^{-\sum_{j=1}^{i} d_{j} \Delta}-d_{i} N_{i},
\end{aligned}
$$

for $i=2, \ldots, \infty$. This model is identical to Eq. (12) when one restricts $d_{i}=d$ by setting $\alpha=0$. Because the models are nested we can test whether allowing $\alpha \neq 0$ gives a significant improvement of the fit. The program that we developed to fit this model to CFSE data is publicly available as a Mathematica notebook on theory.bio. uu.nl/rdb/CFSE/bmb05.nb; this self-explanatory notebook can be used as a toolbox to fit other CFSE data.

Comparing Fig. 4 with Fig. 3 demonstrates that incorporating different death rates for the different cohorts markedly improves the quality of the fits (especially at low IL-2 concentrations). Using the $F$-test to compare the model with $\alpha=0$ statistically to the model with $\alpha \neq 0$, we found a significant improvement of the fit for a death rate increasing with the division number, i.e., $\alpha>0$ see Table 2 , for the three lowest IL-2 concentrations (all three $p<0.001$ and $p=0.1$ for 
(a)

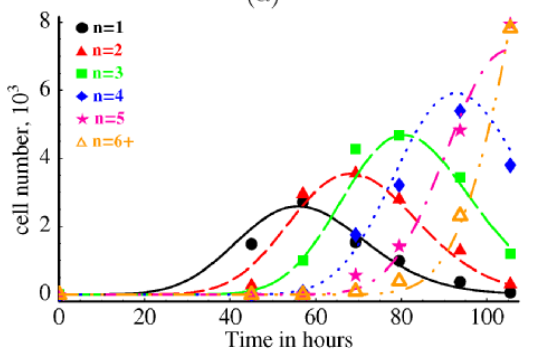

(c)

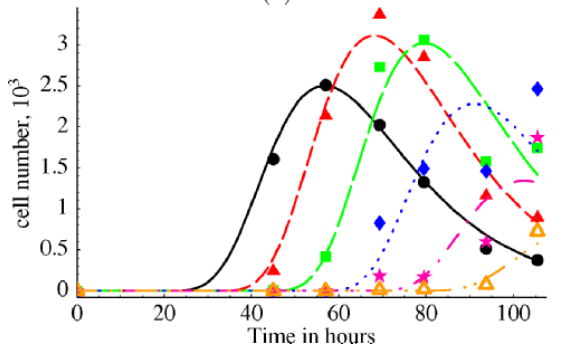

(b)

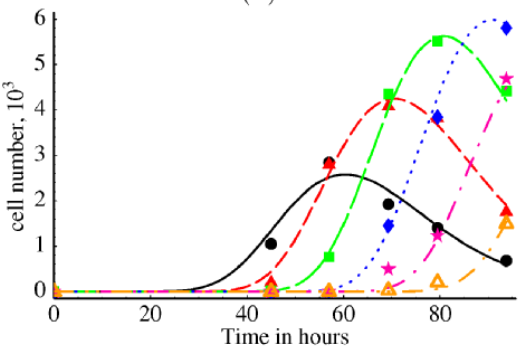

(d)

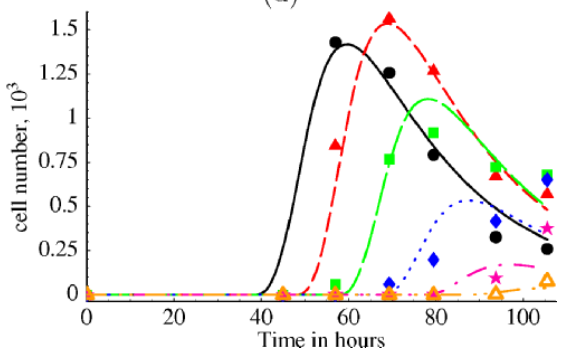

Fig. 4 Division number cohorts of the heterogeneous Deenick et al. (2003) model fitted to CFSE data for IL-2 concentrations of $50 \mathrm{U} / \mathrm{ml}$ (a), $5 \mathrm{U} / \mathrm{ml}$ (b), $2.5 \mathrm{U} / \mathrm{ml}$ (c), and $1.25 \mathrm{U} / \mathrm{ml}$ (d). Parameter values are given in Table 2 .

IL-2 $=50 \mathrm{U} / \mathrm{ml}$ ). The second division cohort in Fig. $4 \mathrm{~d}$ is now higher than the first and the later ones. The first three cohorts for an IL-2 concentration of $2.5 \mathrm{U} / \mathrm{ml}$ now fit the data much better (Fig. 4). The first four cohorts in Fig. 4 also fit the data much better, and only for the highest concentration of IL-2 has it made little difference to add this division dependent death rate. The dependence of the death rate on the division number differs among the different concentrations of IL-2 (Fig. 5b), and is strongest for the lowest IL-2 concentration, intermediate for the intermediate concentrations, and virtually absent for the highest IL-2 concentration. The recruitment functions estimated for the three highest concentrations of IL-2 are fairly similar and symmetric (Fig. 5a). For the lowest IL-2 concentration, the estimated $R(t)$ is asymmetric with a long tail. Experimentally, the time to enter the first S-phase after stimulation was estimated more directly by thymidine incorporation. This data suggested that all four recruitment functions were asymmetric and had fairly similar shapes (Deenick et al., 2003). We therefore think that the three estimated symmetric $R(t)$ functions are not realistic, and are an artifact of the simplifying assumption of the DGH model that all divisions after the first have the same cell cycle time, forcing the solutions of the later cohorts to all have identical shapes.

Despite the potential problem with $R(t)$, the model describes the data reasonably well, and we computed confidence intervals on the parameters by bootstrapping (Efron and Tibshirani, 1986). The results are summarized in Fig. 6 and Table 2 , which shows parameter values and $95 \%$ confidence intervals indicated by error bars as a function of the IL-2 concentration. The first three panels in Fig. 6 
(a)

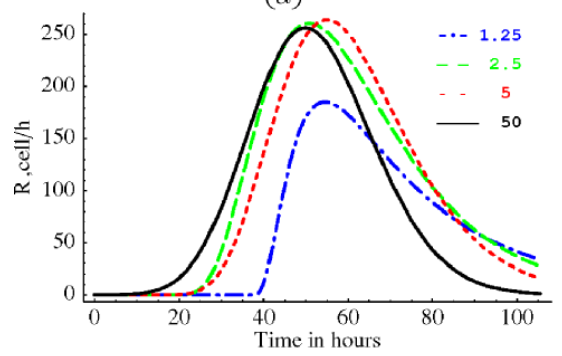

(b)

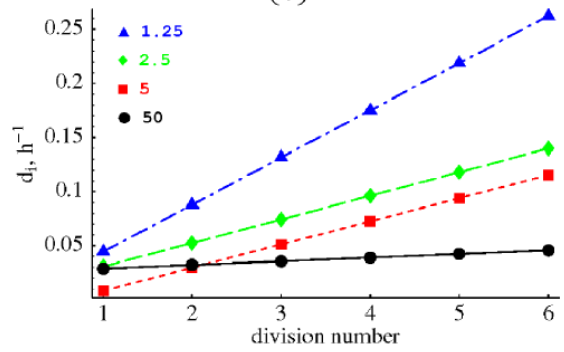

(c)

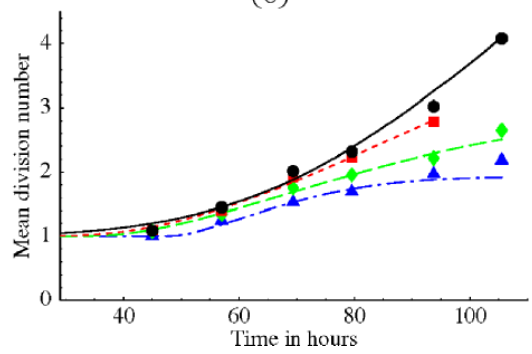

Fig. 5 Recruitment functions (a), death rates (b), and mean division numbers $\widehat{\mu}$ for the four IL2 concentrations fitted with the model of Eq. (13). The solid line depicts an IL-2 concentration of $50 \mathrm{U} / \mathrm{ml}$, the short-dashed line $5 \mathrm{U} / \mathrm{ml}$, the long-dashed line $2.5 \mathrm{U} / \mathrm{ml}$, and the dash-dotted line $1.25 \mathrm{U} / \mathrm{ml}$. Parameters are given in Table 2 .

describe the parameters underlying how $R(t)$ changes with the IL-2 concentration. The average time and its standard deviation to complete the first division (Fig. 6a) increase when the IL-2 concentration is reduced. The total area under the curve, $C$, which is the number of cells that are ultimately recruited to divide is somewhat lower for the lowest concentration of IL-2, which is in agreement with the earlier analysis of this data (Deenick et al., 2003). The cell cycle time $\Delta$ in Fig. $6 \mathrm{~d}$ hardly changes with the IL-2 concentration. The death rate of cells that have undergone one division seems to increase when the IL-2 concentration is reduced (Fig. 6e), and the outlier at an IL-2 concentration of $5 \mathrm{U} / \mathrm{ml}$, was to be expected from the experimental error discovered in Fig. 1. As discussed above, the dependence of the death rate on the division number increases strongly when the IL-2 concentration is reduced (Fig. 6f). In the previous fitting of this data by Deenick et al. (2003), the cell cycle time depended on the IL-2 concentration. We think that this is now partly explained by the increased death at higher division numbers at the low concentrations of IL-2. This illustrates that one should interpret these fits causiously because there are various possibilities providing very reasonable fits to the data. Confidence intervals therefore seem essential, because they can provide an indication of the validity of the parameter estimates. More importantly, we need additional measurements to distinguish between models.

Above we have noted that the cell cycle time estimated from the slope of the mean division number in Table 1 is about twofold longer than that estimated by 
(a)

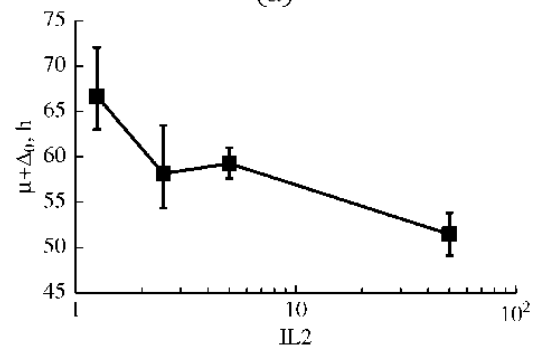

(c)

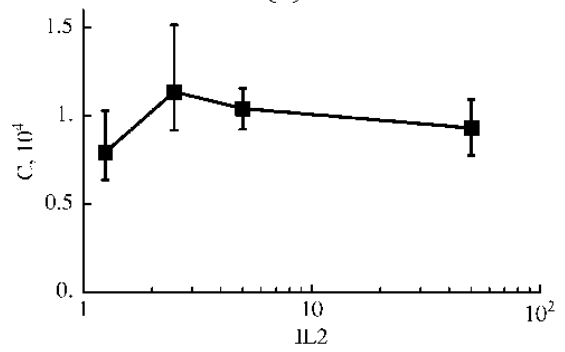

(e)

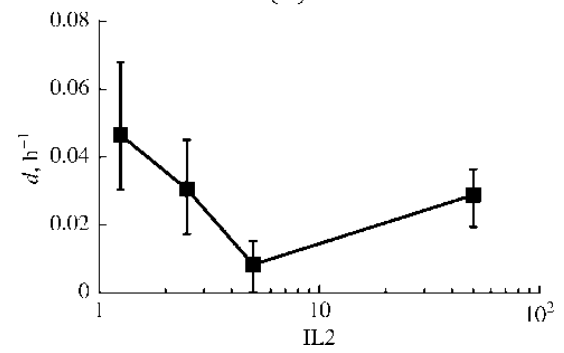

(b)

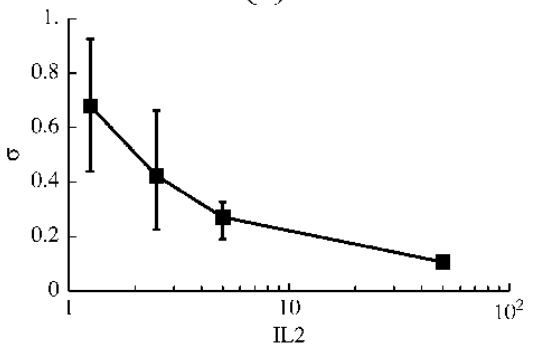

(d)

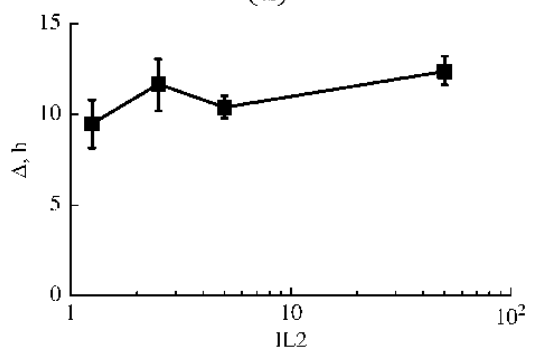

(I)

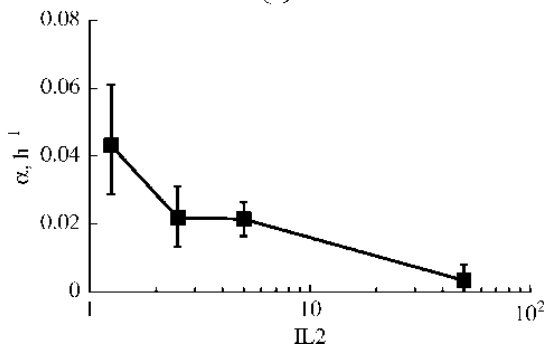

Fig. 6 Parameters as a function of the IL2 concentration. Average time to complete first division $\mu+\Delta_{0}$ (a), standard deviation $\sigma$ in $R(t)$ (b), total number of cell recruited into division (c), cell cycle time $\Delta(\mathrm{d})$, death rate $d$ of cells having completed one division (e), and slope of death rates $\alpha$ (f). Error bars denote $95 \%$ confidence intervals as obtained by bootstrapping the residuals with 500 simulations (Efron and Tibshirani, 1986).

fitting the DGH model in Table 2. What would be responsible for these different estimates? Part of the answer is provided by Eq. (10), which says that the mean division number, $\widehat{\mu}$, approaches the asymptote, $\widehat{\mu}=p t$, only after an initial transient depending on the division rate $p$ (De Boer and Perelson, 2005). For an estimated divison rate of $20 \mathrm{~h}$, it will take approximately $60 \mathrm{~h}$, i.e., three divisions, for $\widehat{\mu}$ to be within $5 \%$ of its asymptoptic value. Indeed, plotting $\widehat{\mu}$ in the extended DGH model with the fitted parameter values together with the mean division numbers from the data reveals that most of the data points fall within this transient (Fig. 5c). The linear regression line through the data will therefore have a smaller slope than the asymptote, and one is overestimating the cell cycle time when 
estimating it from the mean division numbers only (De Boer and Perelson, 2005). Additionally, we have shown previously that this transient can become even longer in more complicated models in which the first division takes longer than the later divisions (De Boer and Perelson, 2005), which is the case for the T cells studied here. Finally, note that for the lowest IL-2 concentration the mean division number never approaches the asymptote corresponding to the cell cycle time. The reason for this is that the death rate is so high that the population of divided cells is contracting (Table 1), while the recruitment function continues to deliver novel cells in the first division class. When the cells having completed several divisons die out, the fraction of cells having completed only one division increases, and $\widehat{\mu}$ decreases (Fig. 5c: dash-dotted line). Because of these long transients one should recognize that estimates of division times determined by linear regression of the mean division numbers may be inaccurate, especially for data sets collected over short time intervals (De Boer and Perelson, 2005). Thus, one should consider estimates obtained this way only as upper bounds.

\section{Future models}

We have suggested two extensions of the simple DGH model (Deenick et al., 2003). First, the death rate seems to depend on the division number, and second the model needs to reconcile the observed asymmetric recruitment in the thymidine data (Deenick et al., 2003) with the apparently symmetric division cohorts later on (Fig. 4). We have recently analyzed the shape of the division cohorts by fitting them to various distributions, and observed that cohorts indeed tend to become more symmetric with the division number (results not shown). To change the shape of the division cohorts one has to relax the assumption of completely deterministic later divisions in the DGH model. In the original Deenick et al. (2003) paper it was already noted that the variability of division times seemed to increase when the IL-2 concentration was reduced, and that stochasticity in the later divisions would be required in the future. Additional experimental techniques, like BrdU labeling or measuring cell size and DNA content, are probably required to establish the stochasticity of later divisions and whether parameters change with time or the division number. As a first theoretical approach we are currently working on two extensions of the DGH model (Deenick et al., 2003) that both allow for stochastic recruitment into the later divisions.

\subsection{Smith-Martin model}

A standard mathematical model for analyzing the kinetics of the cell cycle is the Smith-Martin (Smith and Martin, 1973) model, which allows for two phases of the cell cycle. Cells in the "A"-state are approximately in the G1 (or G0) phase of the cell cycle, and are recruited stochastically to divide, i.e., to enter the so-called "B"-phase. The B-phase approximately corresponds to the S, G2, and M phases of the cell cycle and has a fixed total duration $\Delta$. After completing the "deterministic" B-phase, a cell delivers two daughter cells in the "stochastic" A-state from which the cells may be recruited for another round of division (Smith and Martin, 1973). 
Importantly, cells differing in the length of the cell cycle typically differ in the length of the A-state. Once triggered to divide, cells typically proceed through the deterministic B-phase at approximately the same speed (Smith and Martin, 1973). The Deenick et al. (2003) model presented above is a special case of the SmithMartin model that is obtained when $\lambda \rightarrow \infty$, i.e., when the length of the stochastic A-state is very short. Due to its deterministic B-phase the Smith-Martin model prevents the too rapid progression through cell cycle cascades that one observes in ODE models (De Boer and Perelson, 2005). Cells in the A-state and B-phase have death rates $d_{\mathrm{A}}$ and $d_{\mathrm{B}}$, respectively, and cells leave the A-state at rate $\lambda$. Using the same recruitment function, $R(t)$, for the number of cells completing the first division, and entering the A-state of the next division, the Smith-Martin model can be formulated as a set of delay differential equations for the cells in the Astate of the various division indexes. The model is

$$
\begin{aligned}
\frac{\mathrm{d} A_{1}(t)}{\mathrm{d} t}= & R(t)-\left(\lambda+d_{\mathrm{A}}\right) A_{1}(t), \quad A_{1}(0)=0, \\
\frac{\mathrm{d} A_{i}(t)}{\mathrm{d} t}= & 2 \lambda A_{i-1}(t-\Delta) \mathrm{e}^{-d_{\mathrm{B}} \Delta} \\
& -\left(\lambda+d_{\mathrm{A}}\right) A_{i}(t), \quad A_{i}(0)=0, \quad i=2, \ldots, \infty,
\end{aligned}
$$

where the number of cells in the B-phase of each division class are

$$
B_{i}(t)=\lambda \int_{0}^{\Delta} A_{i}(t-\tau) \mathrm{e}^{-d_{\mathrm{B}} \tau} \mathrm{d} \tau, \quad i=1, \ldots, \infty,
$$

where the total number of cells in each cohort, $N_{i}(t)=A_{i}(t)+B_{i}(t)$, can be compared to the DGH model. One can use an $F$-test for nested models to test whether the Smith-Martin model gives a significantly better fit to the data. The major advantage of fitting CFSE data with the Smith-Martin model is that asymmetric recruitment functions, $R(t)$, are compatible with symmetric cohort profiles later on [Ganusov et al., in preparation].

\subsection{General probability distributions}

A great deal of experimental work over several years examined the independence of the growth and death rates of cells undergoing a cascade of cell divisions. The nature of the birth and death probability distributions, the inheritance of parameters from parent to daughter cells, and parameter values could all be linked to the time since cell stimulation, or the number of divisions cells have completed (Gett and Hodgkin, 1998, 2000; Deenick et al., 2003; Hasbold et al., 2004). The Hodgkin laboratory has therefore been developing very general numerical tools in which division and death can independently be described by arbitrary probability distributions. These tools are used intensively to fit data, test assumptions of the numerical model, and explore further measurements that may constrain the fitting of the model to the data. An earlier version of this numerical model, i.e., the 
Deenick et al. (2003) model, was reformulated as a mathematical model by Leon et al. (2004) with arbitrary probability distributions for the cell division times.

To provide an idea of the model that is currently being developed [Hawkins, Turner, Van Gend \& Hodgkin, in preparation], we formulate a structured population model (Diekmann et al., 2001; Bernard et al., 2003; Pilyugin et al., 2003) in which the rates of cell division and death are functions of cell age

$$
\frac{\partial n_{i}(t, s)}{\partial t}+\frac{\partial n_{i}(t, s)}{\partial s}=-\left[p_{i}(s)+d_{i}(s)\right] n_{i}(t, s) \quad \text { with } \quad N_{i}(t)=\int_{0}^{\infty} n_{i}(t, s) \mathrm{d} s,
$$

where $n_{i}(t, s)$ is defined as the time-density of cells of age $s$, having completed $i$ divisions at time $t$, and with boundary conditions

$$
\begin{aligned}
& n_{1}(t, 0)=R(t) \\
& n_{i}(t, 0)=2 \int_{0}^{\infty} p_{i-1}(s) n_{i-1}(t, s) \mathrm{d} s, \quad i=2, \ldots, \infty
\end{aligned}
$$

The proliferation rate, $p_{i}(s)$, and the death rate, $d_{i}(s)$, are determined by the underlying probability distributions. Depending on the form of each probability distribution, and the number of different distributions, this model can have few or many parameters.

\section{Discussion}

Fitting the extended DGH model to the data on the dynamics of T cell stimulated under different concentrations of IL-2, suggests that increasing the IL-2 concentration, decreases the time to complete the first division, decreases the death rate, and decreases the dependence of the death rate on the cell division number (Fig. 6). These results differ considerably from those obtained by fitting the orginal DGH model manually to the same data (Deenick et al., 2003). One reason for this difference could be that we here used a log normal recruitment function to fit the data and that previously a normal recruitment function was fitted to the data. Another difference is the dependence of the death rate on the division number in the extended DGH model of Eq. (13). This shows that these parameter estimated have to be interpreted carefully, and that it is important to check different models and test the validity of parameter estimates by computing confidence intervals. Our results have shown that to properly describe the CFSE data with the data on recruitment into first disvion (Deenick et al., 2003), one needs to further extend the DGH model with stochastic recruitment into the later divisions. We have discussed two future models that allow for this.

Another major concern in interpreting CFSE data quantitatively is that the increase in the mean division number is not only determined by the division rate, but is also influenced by the way death is distributed over the cell cycle (Pilyugin et al., 2003; Ganusov et al., 2005). For instance, cell death could occur mainly in the S, $\mathrm{G} 2$ and $\mathrm{M}$ phase of the cell cycle because of the various check points during these 
phases, and death could be rare during the quiescent G1 (or G0) phase. If this is the case, and if the period to complete the $\mathrm{S}, \mathrm{G} 2$ and $\mathrm{M}$ phases of the cell cycle is a small fraction of the total time between divisions, the slope of the linear increase of the mean division number is a composite of the cell cycle time and the death rate of the cells (Pilyugin et al., 2003; Ganusov et al., 2005), and one may overestimate the cell cycle time. Pilyugin et al. (2003) and Ganusov et al. (2005) show that CFSE data contain too little information to estimate how death is distributed over the cell cycle, and conclude that one can only estimate (a) the average cycle time of the cells successfully completing division, and (b) the fraction of cells surviving the cell cycle. However, most biologists seek estimates for the average cell cycle time and the average cell death rate, where the average is taken over all cells. We have shown that for the data under consideration here, i.e., cells that are expanding rapidly, the Deenick et al. (2003) model with a cell cycle that is composed of a single deterministic phase describes the data reasonably well. With this model we have avoided the problem of the distribution of the death rates. These results remain in good agreement with the analysis of Pilyugin et al. (2003) and Ganusov et al. (2005) because the distribution of death rates is most important when when populations are at steady state, or declining, and when most death occurs during a relatively short phase of the cell cycle.

\section{Acknowledgements}

We dedicate this paper to Lee Segel who witnessed the development of this work and our collaboration during the wonderful summers we spent together at the Santa Fe Institute. Lee played an important role not only in discussing science, but also in taking us to the Santa Fe opera, to other musical highlights, on beautiful hikes in the mountains, and to delightful dinners at his home. Our summers in Santa Fe will never be the same. We have lost a dear friend and colleague. We thank the Santa Fe Institute for hosting us to collaborate on our HFSP grant RGP0010/2004. Portions of this work were done under the auspices of the U.S. Department of Energy under contract W-7405-ENG-36, and supported by NIH grants AI28433 and RR06555 (ASP). RdB acknowledges the financial support of NWO grant 016.048.603.

\section{Appendix}

For the Deenick et al. (2003) model we can define the time-density of cells, $n_{i}(t, s)$ that have completed $i$ divisions at time $t$ and have spent time $s$ is the $i$ th division class (see (Pilyugin et al., 2003; Ganusov et al., 2005) for more detail). Then $n_{i}(t, s)$ should satisfy the following PDE:

$$
\frac{\partial n_{i}(t, s)}{\partial t}+\frac{\partial n_{i}(t, s)}{\partial s}=-d n_{i}(t, s), \quad i=1, \ldots, \infty
$$

with boundary conditions

$$
n_{1}(t, 0)=R(t) \quad \text { and } \quad n_{i}(t, 0)=2 n_{i-1}(t, \Delta), \quad i=2, \ldots, \infty,
$$


and the initial condition $n_{i}(0, s)=0 \forall i$. The total number of cells having completed $i$ divisions by time $t$ is $N_{i}(t)=\int_{0}^{\Delta} n_{i}(t, s) \mathrm{d} s$, given that the duration of the $i$ th division is $\Delta$. Equation (18) has a solution, $n_{i}(t, s)=G_{i}(t-s) \mathrm{e}^{-d s}$, where $G_{i}(t)$ is a general function that is found using the boundary conditions. For example, for $i=1, G(t)=R(t)$ and

$$
N_{1}(t)=\int_{0}^{\Delta} R(t-s) \mathrm{e}^{-d s} \mathrm{~d} s .
$$

Similarly, $G_{2}(t)=2 n_{1}(t, \Delta)$, and

$$
\begin{aligned}
N_{2}(t) & =2 \int_{0}^{\Delta} n_{1}(t-s, \Delta) \mathrm{e}^{-d s} \mathrm{~d} s=2 \mathrm{e}^{-d \Delta} \int_{0}^{\Delta} R(t-s-\Delta) \mathrm{e}^{-d s} \\
\mathrm{~d} s & =2 \mathrm{e}^{-d \Delta} N_{1}(t-\Delta) .
\end{aligned}
$$

Continuing this relationship recursively, one obtains Eq. (12) shown in the main text.

The recruitment function, $R(t)$, used for fitting the DGH model to the CFSE data is the non-normalized log-normal distribution

$$
R(t)= \begin{cases}\frac{C}{\sqrt{2 \pi} \sigma\left(t-\Delta_{0}\right)} \times \mathrm{e}^{-\frac{\left(\log \left(t-\Delta_{0}\right)-\log (\mu)\right)^{2}}{2 \sigma^{2}},}, & \text { if } t>\Delta_{0} \\ 0, & \text { otherwise }\end{cases}
$$

where $\Delta_{0}$ is the location parameter, $\sigma$ is the shape parameter and $\mu$ is the scale parameter. Since $\int_{\Delta_{0}}^{\infty} R(t) \mathrm{d} t=C$, the parameter $C$ denotes the total number of divided cells that have entered their second division. Thus, if at $t=0$ there were $N_{0}$ cells present, then the ratio $C / N_{0}$ denotes the fraction of cells that have completed their first division. If the death rate of undivided cells is constant and is independent of time, then the ratio $C / N_{0}$ is proportional to the fraction of cells recruited into the division.

\section{References}

Bernard, S., Pujo-Menjouet, L., Mackey, M.C., 2003. Analysis of cell kinetics using a cell division marker: mathematical modeling of experimental data. Biophys. J. 84(5), 3414-3424.

De Boer, R.J., Perelson, A.S., 2005. Estimating division and death rates from CFSE data. J. Comp. Appl. Math. 184, 140-164.

Deenick, E.K., Gett, A.V., Hodgkin, P.D., 2003. Stochastic model of T cell proliferation: A calculus revealing IL-2 regulation of precursor frequencies, cell cycle time, and survival. J. Immunol. 170, 4963-4972.

Diekmann, O., Gyllenberg, M., Huang, H., Kirkilionis, M., Metz, J.A., Thieme, H.R., 2001. On the formulation and analysis of general deterministic structured population models. II. Nonlinear theory. J. Math. Biol. 43, 157-189.

Efron, B., Tibshirani, R., 1986. Bootstrap methods for standard errors, confidence intervals, and other measures of statistical accuracy. Stat. Sci. 1, 54-77.

Ganusov, V.V., Pilyugin, S.S., De Boer, R.J., Murali-Krishna, K., Ahmed, R., Antia, R., 2005. Quantifying cell turnover using CFSE data. J. Immunol. Methods 298, 183-200. 
Gett, A.V., Hodgkin, P.D., 1998. Cell division regulates the T cell cytokine repertoire, revealing a mechanism underlying immune class regulation. Proc. Natl. Acad. Sci. U.S.A. 95, 9488-9493.

Gett, A.V., Hodgkin, P.D., 2000. A cellular calculus for signal integration by T cells. Nat. Immunol. 1, 239-244.

Hasbold, J., Corcoran, L.M., Tarlinton, D.M., Tangye, S.G., Hodgkin, P.D., 2004. Evidence from the generation of immunoglobulin G-secreting cells that stochastic mechanisms regulate lymphocyte differentiation. Nat. Immunol. 5, 55-63.

Hasbold, J., Gett, A.V., Rush, J.S., Deenick, E., Avery, D., Jun, J., Hodgkin, P.D., 1999. Quantitative analysis of lymphocyte differentiation and proliferation in vitro using carboxyfluorescein diacetate succinimidyl ester. Immunol. Cell. Biol. 77, 516-522.

Kaech, S.M., Ahmed, R., 2001. Memory CD8 ${ }^{+} \mathrm{T}$ cell differentiation: initial antigen encounter triggers a developmental program in naive cells. Nat. Immunol. 2, 415-422.

Leon, K., Faro, J., Carneiro, J., 2004. A general mathematical framework to model generation structure in a population of asynchronously dividing cells. J. theor. Biol. 229, 455-476.

Lyons, A.B., 2000. Analysing cell division in vivo and in vitro using flow cytometric measurement of CFSE dye dilution. J. Immunol. Methods. 243, 147-154.

Milutinović, D., 2004. Stochastic Model of Micro-Agent Populations. PhD Thesis, Institute for Systems and Robotics, Instituto Superior Tecnico, Lisbon, Portugal.

Mosmann, T.R., Bond, M.W., Coffman, R.L., Ohara, J., Paul, W.E., 1986. T-cell and mast cell lines respond to B-cell stimulatory factor 1. Proc. Natl. Acad. Sci. U.S.A. 83(15), 5654-5658.

Nordon, R.E., Nakamura, M., Ramirez, C., Odell, R., 1999. Analysis of growth kinetics by division tracking. Immunol. Cell. Biol. 77(6), 523-529.

Pilyugin, S.S., Ganusov, V.V., Murali-Krishna, K., Ahmed, R., Antia, R., 2003. The rescaling method for quantifying the turnover of cell populations. J. Theor. Biol. 225, 275-283.

Revy, P., Sospedra, M., Barbour, B., Trautmann, A., 2001. Functional antigen-independent synapses formed between T cells and dendritic cells. Nat. Immunol. 2, 925-931.

Smith, J.A., Martin, L., 1973. Do cells cycle? Proc. Natl. Acad. Sci. U.S.A. 70, 1263-1267.

Van Stipdonk, M.J., Lemmens, E.E., Schoenberger, S.P., 2001. Naive CTLs require a single brief period of antigenic stimulation for clonal expansion and differentiation. Nat. Immunol. 2, $423-429$.

Veiga-Fernandes, H., Walter, U., Bourgeois, C., McLean, A., Rocha, B., 2000. Response of naive and memory $\mathrm{CD} 8^{+} \mathrm{T}$ cells to antigen stimulation in vivo. Nat. Immunol. 1, 47-53.

Zhang, X.W., Audet, J., Piret, J.M., Li, Y.X., 2001. Cell cycle distribution of primitive haematopoietic cells stimulated in vitro and in vivo. Cell. Prolif. 34(5), 321-330. 